
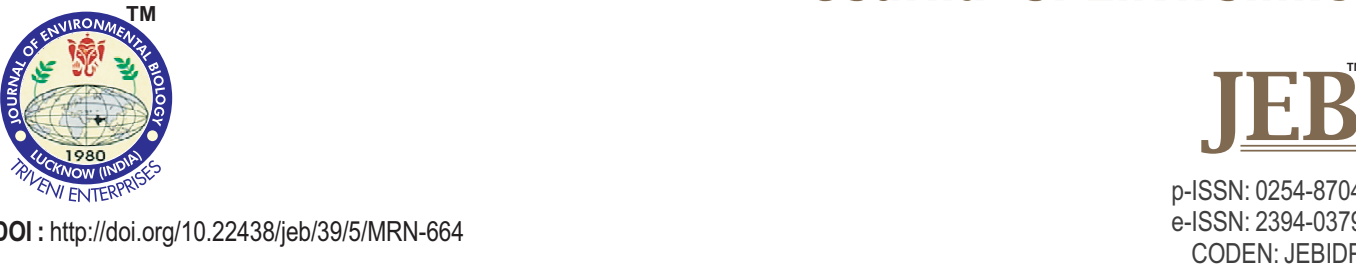

\title{
Protective effect of folic acid against arsenic induced weight loss during gestation, lactation and postnatal growth in mice
}
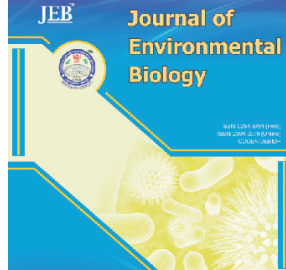

Authors Info

Sh. Mirfakhraie', Z.J. Masoumeh ${ }^{2 *}$, K. Mojtaba ${ }^{3}$ and B. Mohammad ${ }^{3}$

1 Department of Entomology, Urmia University, Urmia, West Azarbayjan, 57561 51818, Iran

${ }^{2}$ Department of Anatomy, Urmia University of Medical Sciences, Urmia, West Azarbayjan, 57561 15111, Iran

${ }^{3}$ Department of Pathology, Urmia University of Medical Sciences, Urmia, West Azarbayjan,

57561 15111, Iran

${ }^{*}$ Corresponding Author Email : ms_zirak@yahoo.com

Key words

Arsenic toxicity

Blood-brain barrier

Folic acid

Gestation

Postnatal growth

\section{Publication Info}

Paper received : 06.06.2017

Revised received : 01.11.2017

Re-revised received : 13.02 .2018

Accepted : 29.03 .2018

\section{Abstract}

Aim : The present study aimed to determine weight loss in sodium arsenate exposed pups during gestation, lactation and postnatal growth. Additionally, the protective role of folic acid was also assessed against impaired growth caused by arsenic during three developmental stages.

Methodology : Twenty pregnant albino mice were divided into four groups and received the following treatment in their drinking water from gestational day one (G1): control group, $1 \mathrm{mgl}^{-1} \mathrm{As} 1 \mathrm{mg} \mathrm{l}^{-1} \mathrm{As}+25 \mathrm{mg}$ $\mathrm{kg}^{-1} \mathrm{FA}(\mathrm{As}+\mathrm{FA})$ by ip once a week during gestation and lactation and $25 \mathrm{mg} \mathrm{kg}^{-1}$ folic acid (FA) groups. After weaning, the litters were directly exposed to arsenic and folic acid with the same dosage.

Results : Exposure to arsenic exhibited a significant $(P<0.01)$ growth impairment in the weight of offspring during lactation $\left(1^{\text {st }}-3^{\text {rd }}\right.$ week) and $22-56$ ds postnatal (PN) in the As-treated group. The administration of FA through gestation and lactation by dams after 2 months could significantly $(P<0.01)$ prevent As-induced weight loss in the developmental stages. Treatment with FA resulted in significantly decrease $(P<0.05)$ of AS level in liver and brain.

Interpretation : These observations indicate that vitamin administration could prevent growth impairments of arsenic exposure.

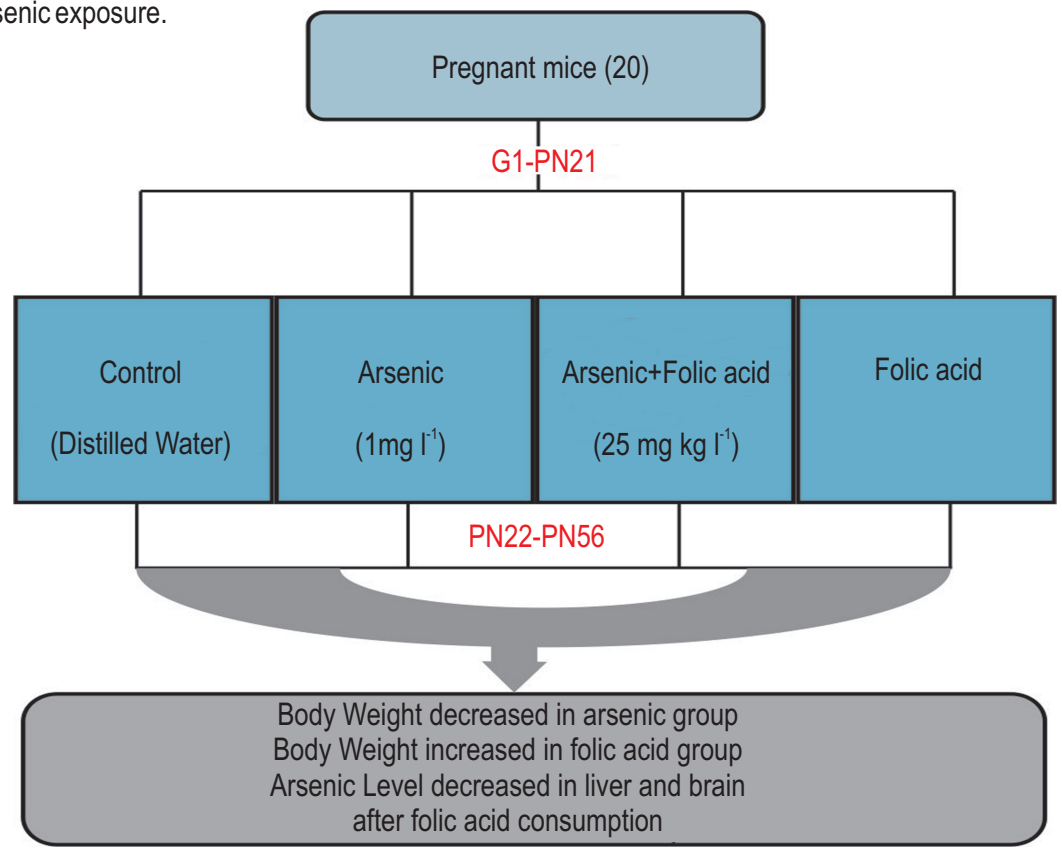




\section{Introduction}

Climate Chronic arsenic exposure causes a wide range of health issues such as cancer, developmental toxicity, cardiovascular and brain dysfunction in children and young adults. This old and naturally occurring poison has a long history of toxicity in food, water, soil and air (Hughes et al., 2011). It is widely present in herbicides such as organoarsenical (Chen et al., 2008). Micronutrients supplements, such as folate, have protective role for decreasing some of the developmental toxicity of arsenic. Folic acid is a source for generation of methionine, facilitates the methylation process and increases arsenic secretion in urine and relieve its toxicity (Hall and Gamble, 2012). It has been reported that maternal pretreatment with folic acid can prevent fetal death and intrauterine growth restriction induced by lipopolysaccharide in mice. Protective mechanism of folic acid is related to anti-inflammatory effects and reduction of interlukins and cytokins in amniotic fluid (Zaho et al., 2013). Another animal study established that folate deficiency diet during lactation resulted in decreased body weight of offspring on third week of lactation compared to normal diet consumption in rats (Berrocal-Zaragoza et al., 2014).

Mice treated with $10 \mathrm{ppb}$ arsenic in their drinking water, during the embryonic and postnatal period, induced significant impairment of growth in the early period of development after birth, as a result of decreased nutrients in the dam's breast milk (Kozul-Horvath et al., 2012). The beneficial effects of breast feeding have been previously demonstrated, but the nutritional quality of donor breast milk is important. Other investigation has been revealed that low levels of arsenic were detected in an analysis of breast milk samples and urine from infants whose mothers lived in areas with high exposure to arsenic. Although arsenic passes through the milk, it has been indicated that breast feeding protects the infant from the hazards of arsenic (Fängström et al., 2008). There are numerous contradictory results on the effects of exposure to arsenic early in life. However, it is uncertain whether breast feeding leads to reduced infant exposure to arsenic.

Recent studies have focused on the protective effects of antioxidants against As-induced organ toxicity. Folic acid is a water-soluble antioxidant that influences the metabolism of arsenic via methylation. This vitamin plays an important role in growth, development and cell function. Therefore, folic acid is required for fetal growth and contributes highly to both prenatal and postnatal development ((Liu et al., 2016). In this study, for the first time, growth impairment due to arsenic exposure was examined in three developmental periods from postnatal days 1-56. The protective effect of folic acid against arsenic toxicity has also been reported.

\section{Materials and Methods}

Animal study : Twenty female BALB/C albino mice (10 weeks old, $30-70 \mathrm{~g}$ ) were procured from the Urmia University of Medical
Sciences (UMSU) Iran. The animal experiments were started after approval from Ethical committee of (UMSU) Iran. All mice were housed in cages at standard conditions (12/12 hr light and dark and temperature of $20^{\circ} \mathrm{C}$ and $50-60 \%$ humidity).

Experimental design : For the purpose of mating, three female mice were kept overnight with a single male. Following the detection of vaginal plugs, pregnant animals were randomly divided into four equal groups $(n=5)$ and were marked as control, As, As + FA and FA. Dams in the control group received drinking water through gestation (G1-G21 ds) and lactation periods (PN1-PN21). The As group received drinking water treated with $1 \mathrm{mg} \mathrm{l}^{-1}$ As through gestation and lactation. One $\mathrm{mg}$ As was dissolved in one liter of drinking water and solution was prepared fresh each two days.

The dose of one mg was selected based on permissible limit of arsenic ( $\left.0.01 \mathrm{mg} \mathrm{l}^{-1}\right)$ reported by EPA (EPA, 2001). The As + FA group received $1 \mathrm{mgl}^{-1}$ As in their drinking water plus $25 \mathrm{mg} \mathrm{kg}^{-1}$ folic acid (Gefrides et al., 2002) via i.p. injection on the first morning of each week through gestation and lactation. The folic acid group received drinking water plus same dose of folic acid. After weaning (PN21), the litters were directly exposed to water, arsenic and folic acid with the same dosage. The birth weight of the pups was recorded and subsequently, individual weights were taken weekly during lactation $\left(1^{\text {st }}-3^{\text {rd }}\right.$ week). The weight of the pups was then taken weekly until the eighth week of life, up to day 56 (PN56).

Estimation of arsenic content : Froze liver and brain $(50 \mathrm{mg})$ were homogenized in $2.0 \mathrm{ml}$ of distilled water then mixed with one $\mathrm{ml}$ of three normal solforic acid overnight. Tissues were digested in microwave for $10 \mathrm{~min}$ in a $10 \mathrm{ml}$ test tube and finally mixed with sodium tetrahydroborate at $\mathrm{pH} 2$ to volatile arsin generation. Arsenic content was measured by automicabsortion spectrophotometer (Perkinelmer $900 \mathrm{H}$ ) at $193.3 \mathrm{~nm}$ wavelength and compared with five different concentrations of standard samples.

Statistical analysis : The experimental data were analyzed using the Statistical Package for the Social Sciences (SPSS) version 16.0. The mean and standard error of the mean (SEM) were calculated for the weight. ANOVA was applied to assess the significance of the difference in birth weight as well as comparing the mean of arsenic level among the groups. A repeated measure ANOVA was applied to assess the difference in the mean weight through the lactation period (PN1-PN21) and after weaning (PN22PN56). A P value of 0.05 was considered statistically significant.

\section{Results and Discussion}

The number of offspring was 6-10 per dam in the control and experimental groups. The total number of litters and average weight in the four groups were as follows: 35 litters in the control group (1.1286 $\pm 0.12735 \mathrm{~g}), 33$ litters in the As treated group $(1.0576 \pm 0.11734 \mathrm{~g}), 34$ in the As + FA treated group (1.6824 \pm $0.12903 \mathrm{~g})$ and 37 in FA treated group $(1.72341 \pm 0.12651)$. In 


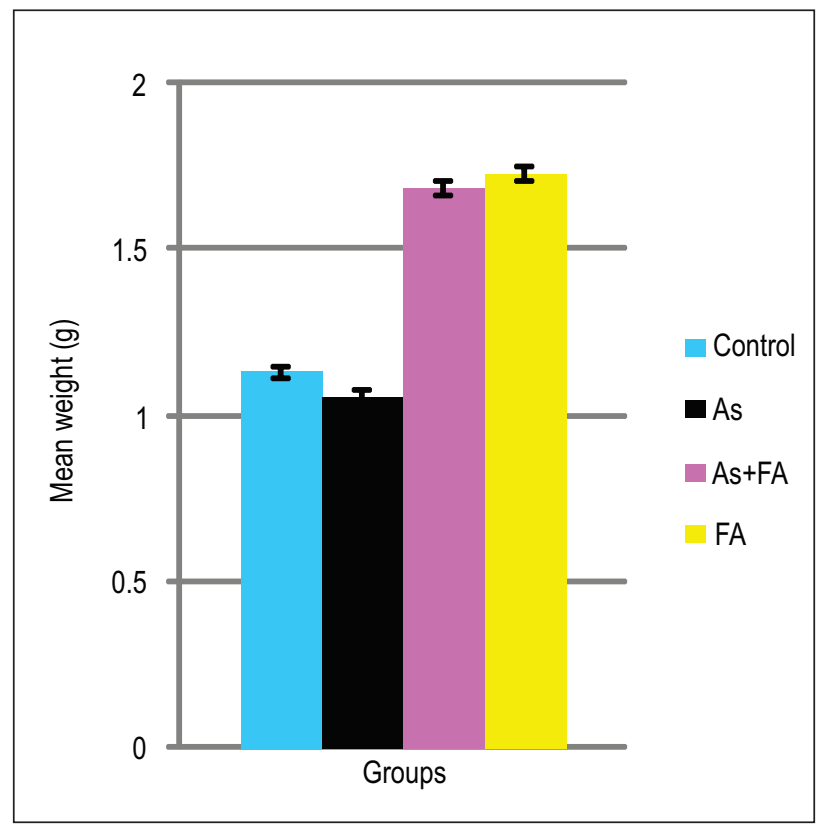

Fig. 1 : Comparison of birth weight of litter. All values are expressed as mean \pm SE (SEM). The mean difference between As and control group NS-not significant at $P<0.05$. ANOVA showed a statistically significant increase in the mean litter weight in As+FA and FA groups compared to control and $A$ s at $P<0.01$

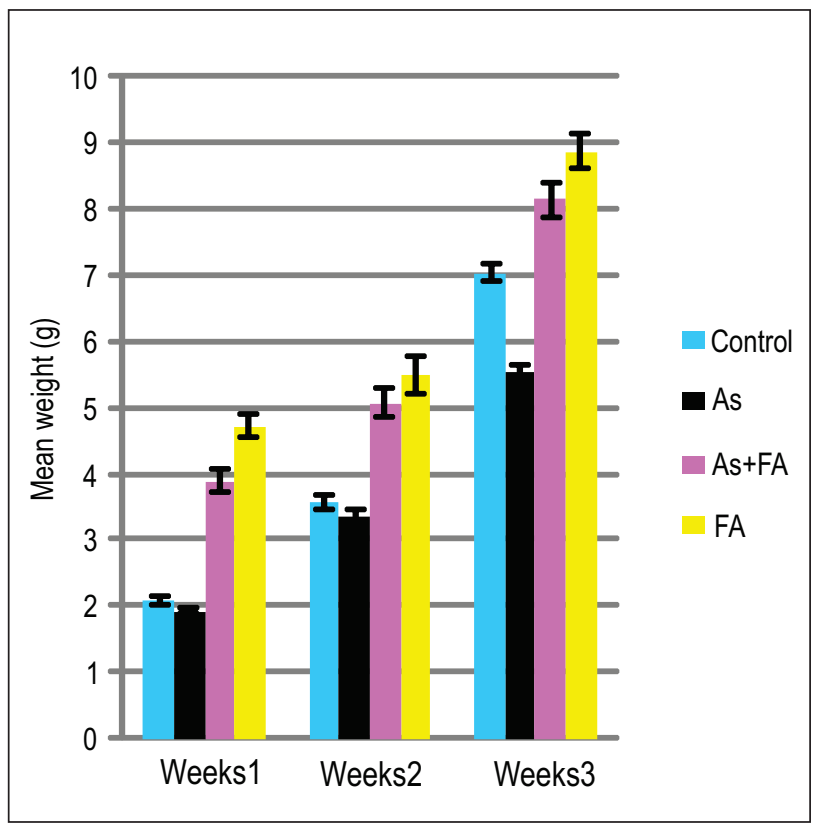

Fig. 2 : Comparison of weight of litter during the lactation period. All values are expressed as mean \pm SEM. A repeated measure of ANOVA showed a statistically significant decrease in the mean litter weight in all groups at $P<0.01$. There was a significant increase in the body weight of litters in both $A s+F A$ and $F A$ groups at $P<0.01$ comparison with the control group, there was no significant difference in the birth weight of pups in the As group. When the mean birth weight of the As + FA and FA groups were compared with the control, the birth weight in the litter seemed to increased. In addition, the difference of the mean litter weight of the As +FA and FA groups were statistically significant when compared to control and As groups (Fig. 1).

The present finding is consistent with the previous studies Ramsey et al. (2017) reported that gestational arsenic exposure to $0.1 \mathrm{mg} \mathrm{I}^{-1}$ had no significant effect on offspring weight. In an another study the mean fetal body weight was not affected by As exposure (1.5, 3 and $4.5 \mathrm{mg} \mathrm{kg}^{-1}$ day $\left.^{-1}\right)$ between gestation day 8 until birth. Significant growth deficits only have been observed with a high dose of arsenic exposure during embryonic period in a mouse model (Gardner et al., 2013). The administration routes of inorganic arsenic are important in determining the adverse developmental effects. Fetal malformations and growth deficiencies were reported when pregnant rats and mice were iv or ip injected with inorganic As during early gestation, as it could be taken up by the blood vessels directly (DeSesso, 2001). The filtration role of placenta is related to fetal-maternal barrier of this organ, which reduces the passage of harmful environmental contaminants (Caserta et al., 2013). The offspring in the As + FA group showed a significant increase in weight compared with the As group. The mechanisms associated with the effect of FA on growth are not fully understood. Studies have suggested that hepatic insulin-like factor 2 (IGF2) expression was unregulated by injecting $150 \mu \mathrm{g} F A$ into fertile eggs on E11, therefore, embryo

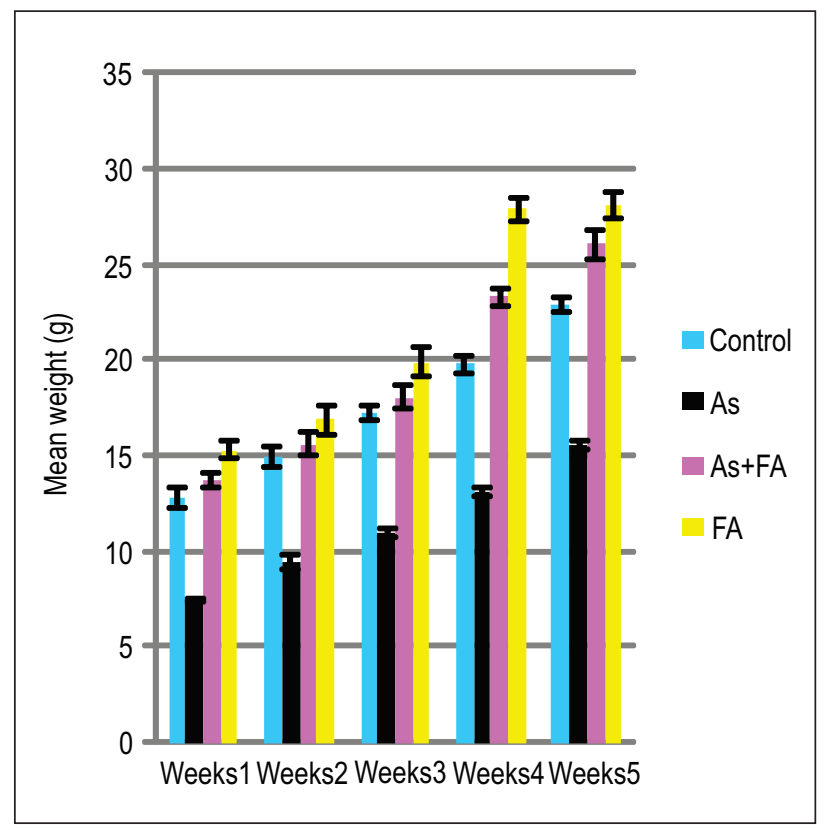

Fig. 3 : Comparison of weight of litter during postnatal days $22-56$. All values are expressed as mean \pm SEM. A repeated measure of ANOVA showed statistically significant decrease in the mean litter weight in all groups at $P<0.01$. There was a significant increase in the body weight of litters in both $A s+F A$ and $F A$ groups at $P<0.01$ 
growth has a positive relationship with hepatic IGF2 expression, cell proliferation and DNA methylation (Liu et al., 2015). One carbon transfer component like folate can transfer from mother to fetus blood circulation and cause cell proliferation, increase of protein mass and fetal growth (Kalhan, 2016).

A decrease in body weight continued throughout the lactation period until weaning at day 21 in the As group. Prominent weight loss started at the end of second week. A repeated measure of ANOVA showed a statistically significant $(P<0.01)$ decrease in the mean litter weight compared with the control group. There was a statistically significant difference in the mean body weight during lactation between the folate groups compared to the control and As groups. There was a marked increase at the end of the lactation period in the folate groups when compared with the As group. However, weight gain in the As group was significantly lower compared with the other three groups (Fig. 2). Therefore, inorganic As is extremely toxic and has negative effects, including decreased body weight of the offspring through the lactation period (PN1-PN21). Growth impairment has been reported in a similar mouse study with lower and much higher doses of exposure (Kozul-Horvath et al., 2012). Epidemiological studies have reported low concentrations of arsenic in breast milk samples of women living in areas prone to arsenic in water. $A$ recent report also suggests that arsenic exposure via breast milk was low, because breast barrier can limit the transfer of arsenic into the mother milk (Carignan et al., 2016). A review of 18 published studies since 2000 to 2016 have reported the highest concentration of arsenic $\left(149 \mu \mathrm{gl}^{-1}\right)$ in West Bengal, India, which was much lower in urine with a mean concentration of $438 \mu^{-1}$ (Rebelo and Caldas, 2016). Although a low level of As is detected in breast milk, it has been reported that there was a decrease in the nutrient content of dam's breast milk, specifically in the triglycerides, which play a role in growth deficits (Kozul-Horvath et al., 2012). A recent study suggests that a daily intake of folic acid was associated with breast folate concentration (Page et al., 2017). The vitamins in breast milk can protect offspring against the negative potential of arsenic and likely improve growth by increased feeding and efficiency in feed utilization. Dietary folic acid intake significantly increased digestive enzyme such as lipase and tripsin activities, resulting in enhanced feed intake (Sesay et al., 2016). It has been reported that folic acid supplementation increased growth markers such as weight gain, nutritional demands and survival (Barros et al., 2009).

The weight gain of the litters in the folic acid group was highest. In the As treated group, the weight gain was lowest from the $4^{\text {th }}-8^{\text {th }}$ week $(7.44,9.8069,11.0793,13.1103$ and $15.6 \mathrm{~g})$. The weekly i.p. in jection of folic acid $\left(25 \mathrm{mg} \mathrm{kg}^{-1}\right)$ increased the weight of the litters from the $4^{\text {th }}-8^{\text {th }}$ week of life $(13.7437,15.9281$, 18.0781, 21.7375 and $24.1656 \mathrm{~g})$. Litters in the As + FA and FA groups showed a consistent progressive increase in weight gain over the 5- week period. There was a significant increase $(P<0.01)$ in weight gain of folate groups compared with those obtained in the As and control groups. The protective effect of folic acid against the negative effects of arsenic is given in Fig. 3 .

The weight of the litter of the arsenic group decreased in the present study following a direct administration of arsenic in the drinking water five weeks after weaning. Gardner et al. (2013) reported severe health effects of elevated arsenic exposure in children, mainly in the form of growth. Decreased metabolic activity in the liver, muscle, kidney and lungs due to gradual deposition of As could be the main reason for decrease in weight. In present study, accumulation of arsenic was observed in liver and brain tissues. Increase of arsenic in different organs affects the metabolic and functional activities of these organs, which in turn, leads to a weight reduction (Roggeman et al., 2014). It has been reported that even low doses (one $\mu \mathrm{m}$ ) of arsenic can inhibit skeletal myoblast cell proliferation and decrease some protein expression and insulin-like growth factor-1 essential for growth (Liu et al., 2015). In present study, the protective effect of folic acid on growth has been demonstrated. Over past decades, several experiments have been conducted to test the effects of protecting elements on As induced toxicities. The effects of barberine against As induced mitochondrial membrane rupture and decreasing reactive oxygen species (Khodayar, 2016) as well as the role of Curcuma to diminish the renal dysfunction have been reported (Saxena, 2009). The effects of folic acid administration might be due to protection from mutagenic DNA-breakage and tissue damage as well as antioxidant potential againstAs induced free radical products (Acharyya et al., 2015). An earlier study revealed that after supplementation with folic acid for 12 weeks, there was an increase in urinary As, excreted as dimethylarsinic acid and decreased blood As concentration. Folic acid protection againstAs-induced apoptotic progression has been documented in a cell culture study where folic acid supplemented medium reduced As-induced toxicity and apoptosis (Xu et al., 2010). The beneficial role of folic acid in reducing the growth inhibitory effects of As may be due to its antioxidant potential and ability to remove As from the blood by the acceleration of As metabolism and excretion in urine.

Level of sodium arsenate was measured in liver and brain of mice on PN56. The As level was very low in control and folic acid groups compared to As exposure groups (Table 1). This study reveals that weekly injection of folic acid could significantly

Table 1 : Sodium arsenate concentration in liver and brain in four treatment groups

\begin{tabular}{llllc}
\hline Tissues & Control & As & As+FA & FA \\
\hline Liver $\left(\mu \mathrm{g} \mathrm{g}{ }^{-1}\right.$ tissue $)$ & $.00947 \pm .0000375$ & $.022000 \pm .0066833$ & $.014950 \pm .0011000^{\mathrm{a}}$ & $.011000 \pm .0000816^{\mathrm{a}}$ \\
Brain $\left(\mu \mathrm{g} \mathrm{g}^{-1}\right.$ tissue $)$ & $.00768 \pm .0000472$ & $.016500 \pm .0036968$ & $.011925 \pm .0017576^{\mathrm{a}}$ & $.00605 \pm .0000823^{\mathrm{a}}$ \\
\hline
\end{tabular}

All values are expressed as mean \pm SEM . The mean sodium arsenate level was significantly decreased in As+FA and FA groups compared with As group at $P<0.05$ 
decrease the As levels in liver and brain tissues. After ingestion, arsenic is quickly absorbed by the gastro intestinal tract and is transferred to the liver in a short time and then immediately methylated in liver (Rodriguez et al., 2004). Wang et al. (2013) reported the presence of As in brain and liver after subchronic exposure of sodium arsenate. Arsenic concentration in the brain of mice was lower than liver, probably due to the presence of blood-brain barrier. It is obvious that maternal diet has an important role in pre- and post-natal development. Long term exposure to arsenic through drinking water during embryonic, lactation and post-weaning periods induced growth deficits in the offsprings, as well as, increased As accumulation in liver and brain tissues. Folate administration confers significant protection probably by facilitating of As excretion and preventing its accumulation in organs.

\section{Acknowledgment}

This study was supported by funding from Urmia University of Medical Sciences (No; 1523).

\section{References}

Acharyya, N., B. Deb, S. Chattopadhyay and S. Maiti: Arsenic-induced antioxidant depletion, oxidative DNA breakage, and tissue damages are prevented by the combined action of folate and Vitamin B $_{12}$. Biol. Trace. Elem. Res., 168, 122-132 (2015).

Barros, M.M., M.J.T.Ranzani-Paiva, L.E. Pezzato, D.R. Falcon and I.G. Guimaraes: Haematological response and growth performance of Nile tilapia (Oreochromis niloticus L.) fed diets containing folic acid. Aquacult. Res., 40, 895-903 (2009).

Berrocal-Zaragoza, M.I., J.M. Sequeira, M.M. Murphy, J.D. FernandezBallart, S.G. Abdel Baki, P.J. Bergold and E.V. Quadros : Folate deficiency in rat pups during weaning causes learning and memory deficits. Br. J. Nutr., 8, 1323-32 (2014).

Carignan-Courtney, C., R. Karagas- Margaret, D. Gilbert-Diamond, L. Cottingham and L. Kathryn : Contribution of breast milk and formula to arsenic exposure during the first year of life in a US prospective cohort. J. Expo. Sci. Environ. Epidemiol., 26, 452 (2016).

Caserta, D., A. Graziano, G.L. Monte, G. Bordi and M. Moscarini: Heavy metals and placental fetal-maternal barrier: A mini-review on the major concerns. Eur. Rev. Med. Pharmacol. Sci., 17, 2198-2206 (2013).

Chen, Z., Y. Cai, G. Liu, H. Solo-Gabriele, G.H. Snyder and J.L. Cisar: Role of soil-derived dissolved substances in arsenic transport and transformation in laboratory experiments. Sci. Total. Environ., 406, 180-189 (2008).

DeSesso, J.M.: Teratogen update: Inorganic arsenic. Teratology, 64, 170-173 (2001).

EPA: National primary drinking water regulations; Arsenic and clarifications to compliance and new source contaminants monitoring 40 CFR Parts 9, 141, 142, 6975-7066 (2001).

Fängström, B., S. Moore, B. Nermell, L. Kuenstl, W. Goessler, M. Grandér, I. Kabir, B. Palm, S. E.Arifeen and M.Vahter : Breastfeeding protects against arsenic exposure in Bangladeshi infants. Environ. Hlth. Perspec., 116, 963 (2008).

Gardner, R.M., M. Kippler, F. Tofail, M. Bottai, J. Hamadani, M. Grandér, B. Nermell and B. Palm: Environmental exposure to metals and children's growth to age 5 years: A prospective cohort study. Am. J. Epidemiol., 177, 1356-1367 (2013).
Gefrides- Lisa, A., D. Bennett-Gregory and H. Finnell- Richard: Effects of folate supplementation on the risk of spontaneous and induced neural tube defects in Splotch mice. Teratology, 65, 63-69 (2002).

Hall, M.N. and M.V. Gamble : Nutritional manipulation of one-carbon metabolism: Effects on arsenic methylation and toxicity. J. Toxicol., http: Ildx.doi.org/10-1155/2012/595307 (2012).

Hughes, M.F., D. Beck, Barbara, Y. Chen, S. Lewis, Ari and J. Thomas, David : Arsenic exposure and toxicology: A historical perspective. Toxicol. Sci., 123, 305-332 (2011).

Kalhan, S.C.: One carbon metabolism in pregnancy: Impact on maternal, fetal and neonatal health. Mol. Cell. Endocrinol., 435, 48-60 (2016).

Khodayar, M.J., M. Javadipour, E. Keshtzarand and M. Rezaei: Role of berberine against arsenic induced oxidative damage in isolated rat liver mitochondria. J. Environ. Biol., 37, 285-290 (2016).

Kozul-Horvath, C. D., F. Zandbergen, B.P. Jackson, R.I. Enelow and J.W. Hamilton: Effects of low-dose drinking water arsenic on mouse fetal and postnatal growth and development. PloS. One. 7, e38249 (2012).

Liu, S.H., R.S. Yang, Y.P. Yen, C.Y. Chiu, K.S.L. Tsai and C. Kuo: Lowconcentration arsenic trioxide inhibits skeletal myoblast cell proliferation via a reactive oxygen species-independent pathway. PloS One, 10, e0137907 (2015).

Liu, Y., L.Zhi, J. Shen, S. Li, J. Yao and X. Yang: Effect of in ovo folic acid injection on hepatic IGF2 expression and embryo growth of broilers. J.Anim. Sci. Technol., 7, 40 (2016).

Page, R., A. Robichaud, T.E. Arbuckle, W.D. Fraser and A.J. MacFarlane : Total folate and unmetabolized folic acid in the breast milk of a cross-section of Canadian women. Am. J. Clin. Nutr. 105, 11011109 (2017).

Ramsey, K.A., N. L. Alexander, P.D. Sly and R.Zosky: In utero exposure to low dose arsenic via drinking water impairs early life lung mechanics in mice. BMC. Pharmacol. Toxicol., 14, 13 (2017).

Rebelo, F. M. and E.D. Caldas: Arsenic, lead, mercury and cadmium: Toxicity, levels in breast milk and the risks for breastfed infants. Environ. Res., 151,671-688 (2016).

Rodriguez, V.M., L.M. Del Razo, J.H. Limon-Pacheco, M. Giordano, L.C. Sánchez-Peña, E. Uribe-Querol, G. Gutiérrez-Ospina and M.E. Gonsebatt: Glutathione reductase inhibition and methylated arsenic distribution in Cd1 mice brain and liver. Toxicol. Sci., 84, 157-166 (2004).

Roggeman, S., G.D. Boeck, H.D. Cock, R. Blust and L. Bervoets: Accumulation and detoxification of metals and arsenic in tissues of cattle (Bostaurus), and the risks for human consumption. Sci. Total. Environ., 466, 175-184 (2014).

Saxena, P.N., S. Anand, N. Saxena and P. Bajaj: Effect of arsenic trioxide on renal functions and its modulation by Curcuma aromatica leaf extract in albino rat. J. Environ. Biol., 30, 525-531 (2009).

Sesay, D. F., H.M. Habte-Tsion, Q. Zhou, M. Ren, J. Xie, B. Liu, R. Chen and L. Pan: Effects of dietary folic acid on the growth, digestive enzyme activity, immune response and antioxidant enzyme activity of blunt snout bream (Megalobrama amblycephala) fingerling. Aquacult., 452, 142-150 (2016).

Wang, X., J. Zhang, L. Zhao, S. Hu and F. Piao: Effect of subchronic exposure to arsenic on levels of essential trace elements in mice brain and its gender difference. Biometals, 26, 123-131 (2013).

Xu, Y., H. Wang, Y. Wang, Y. Zheng and G. Sun: Effects of folate on arsenic toxicity in Chang human hepatocytes: Involvement of folate antioxidant properties. Toxicol. Lett., 195, 44-50 (2010).

Zhao, M., Y.H. Chen, X.T. Dong, J. Zhou, X. Chen, H. Wang, S.X. Wu, M.Z. Xia, C. Zhang and D.X. Xu : Folic acid protects against lipopolysaccharide-induced preterm delivery and intrauterine growth restriction through its anti-inflammatory effect in mice. PLOS One, 8, e82713 (2013). 